\title{
ENUMERATIVE PROPERTIES OF PLANE CONNECTED $n$-LINES
}

\author{
B. C. WONG
}

1. Introduction. Consider $n$ distinct lines $a_{1}, a_{2}, \cdots, a_{n}$, no two of which are parallel, in a euclidean plane $\phi$. These $n$ lines, together with their $n(n-1) / 2$ points of intersection, some or all of which may be coincident, form a configuration which we temporarily denote by $K$. Any one of the points in $K$, say the point of intersection of the lines $a_{i}$ and $a_{j}$, may be regarded as a virtually non-present intersection of $a_{i}$ and $a_{j}$. Such a point will be called a point of non-connection and will be denoted by $Q_{i j}$. The lines $a_{i}$ and $a_{j}$ are then said to be disconnected or to have virtually no intersection at $Q_{i j}$. Let $d$ be the number of points of non-connection in $K$, where

$$
0 \leqq d \leqq(n-1)(n-2) / 2 \text {. }
$$

The condition expressed by (1) will be explained in $\$ 2$. Any point of $K$, not regarded as a point of non-connection, will be called a point of connection and will be denoted by $P_{i j}$ if it is the intersection of the lines $a_{i}$ and $a_{j}$. If the $d$ points of non-connection in $K$ are taken in such a way that each of the $n$ lines has one point of connection with at least one of the remaining lines, the resulting configuration is called a plane connected $n$-line with $d$ points of non-connection and will henceforth be denoted by $\gamma_{d^{n}}$ or just $\gamma$.

The object of this paper is to derive some of the enumerative properties of $\gamma$. What these properties are will be explained as we proceed. They will all be expressed in terms of $n$ and $d$.

2. The maximum number of points of non-connection. If $d=0$, then all the $n(n-1) / 2$ points in $\gamma_{0}$ are points of connection. We may call $\gamma_{0}{ }^{n}$ an absolutely connected $n$-line. Suppose $d>0$. Obviously no $n-1$ of the $d$ assumed points of non-connection can be on any one line, say $a_{1}$. For, if $n-1$ of them did lie on $a_{1}$, then $a_{1}$ would be disconnected from the remaining lines, and $\gamma$ would not be a connected $n$-line. Let there be $n-2$ such points on $a_{1}$. Then $a_{1}$ is connected with another line, say $a_{2}$, by the point $P_{12}$. If $a_{2}$ is to be connected with a third line $a_{3}$, then no more than $n-3$ of the remaining points of nonconnection can be on $a_{2}$. Similarly, if $a_{3}$ is to be connected with a fourth line $a_{4}$, then $a_{3}$ cannot have on it more than $n-4$ of the remaining $d-(n-2)-(n-3)$ points of non-connection. Continuing this proc- 
ess, we find that $d$ cannot exceed the number

$$
\begin{aligned}
D & =(n-2)+(n-3)+\cdots+1 \\
& =(n-1)(n-2) / 2 .
\end{aligned}
$$

If $d=D$, we say that $\gamma_{D}^{n}$ is an open $n$-line; and if $d>D, \gamma_{d}^{n}$ is not a single connected $n$-line and is said to be composite. Unless the contrary is stated, we shall suppose that $0 \leqq d \leqq D$, and that no more than $\nu(2 n-\nu-3) / 2$, which is the sum of the first $\nu$ terms in (2), of the $d$ assumed points of non-connection can be on any $\nu$ of the $n$ lines of $\gamma_{d^{n}}$.

The maximum value $D$ of $d$ may be obtained in other ways. For example, suppose an $n$-line such that each of the points in which one of the $n$ lines meets the remaining $n-1$ lines is a point of connection and that each of the points in which the $n-1$ remaining lines meet, two by two, is a point of non-connection. Then the total number of points of non-connection is the same as that given by (2).

The number of points of connection in a non-composite $\gamma_{d}^{n}$ is always

$$
M=n(n-1) / 2-d .
$$

For an open $n$-line, that is, for the case $d=D$, we have $M=n-1$.

3. Adjacent and non-adjacent points of $\gamma$. Two points of connection, $p_{i j}, p_{i k}$, on the same line $a_{i}$, are said to be adjacent if there is no third point of connection, $P_{i l}$, on $a_{i}$ separating them. If they are separated by one or more points of connection on $a_{i}$, they are said to be non-adjacent. Two points of connection, such as $P_{i j}, P_{k l}$, which are not on any one line, are said to be opposite; they are also nonadjacent.

A point $P_{i j}$ (or a point $Q_{i j}$ ) which is adjacent to four points of connection, two on $a_{i}$ and two on $a_{j}$, is called an inner point of connection (or of non-connection). If it is adjacent to less than four points of connection, it is called an outer point of connection (or of non-connection).

4. Transformation of $n$-lines. We assume that a given $n$-line, in which we may suppose that no three or more lines are concurrent, may be transformed into another by the following process: changing some or all of the $d$ points of non-connection in the given $n$-line into points of connection and an equal number of points of connection into points of non-connection so that the number $d$ of points of nonconnection is preserved. In particular, we may transform any given $n$-line in which some or all of the $d$ points of non-connection are inner points into another in which all the $d$ points of non-connection are 
outer points. For purposes of enumeration we shall use the transformed $n$-line.

5. Segments. The portion of any line in $\gamma_{d^{n}}$ included between two adjacent points of connection is called a closed segment belonging to $\gamma_{d}{ }^{n}$. The term "closed segment" may sometimes be replaced by "pair of adjacent points of connection." That portion of a line issuing from a point of connection and extending indefinitely without passing through another point of connection may be called an open segment.

The number of closed segments in $\gamma_{d}^{n}$ can be easily obtained. First, let us consider an absolutely connected $n$-line $\gamma_{0}{ }^{n}$. Since there are $n-1$ points of connection on each line of $\gamma_{0}{ }^{n}$ and therefore $n-2$ closed segments, the total number of closed segments is $n(n-2)$. Suppose now we remove one of the points of connection, say $P_{i j}$, that is, change it into a point $Q_{i j}$ of non-connection. If $P_{i j}$ is an inner point, it is common to four closed segments, two on $a_{i}$ and two on $a_{j}$. After the removal or change, the four closed segments coalesce into two. If it is an outer point, it may be common to two closed segments, one on $a_{i}$ and one on $a_{j}$, and, after its removal, these two segments are no longer closed; or it may be common to three closed segments, one on $a_{i}$ (or $a_{j}$ ) and two on $a_{j}$ (or $a_{i}$ ) and, after its removal, the one segment becomes open and the other two coalesce into one. In all these three possible cases, the removal of one point of connection diminishes the number of closed segments by two. Therefore, the number of closed segments or pairs of adjacent points of connection in $\gamma_{d}^{n}$ is

$$
I=n(n-2)-2 d .
$$

6. Pairs of non-adjacent points of connection. We now calculate the number $T$ of pairs of non-adjacent points of connection in $\gamma d^{n}$. There are $M(M-1) / 2$, where $M$ is given by (3), pairs of points, both adjacent and non-adjacent, of connection; and if we subtract from this the number $I$, given by (4), of pairs of adjacent points of nonconnection, we have the desired number

$$
\begin{aligned}
T & =M(M-1) / 2-I \\
& =n(n-2)\left(n^{2}-9\right) / 8-d(n+2)(n-3) / 2+d(d-1) / 2 .
\end{aligned}
$$

7. Circuits. Let us call a closed circuit or just a circuit any simple closed polygon formed by three or more adjacent segments of $\gamma^{n}$. On a circuit the number of points of connection is equal to the number of segments. An open $n$-line $\gamma_{D}{ }^{n}$ has no circuit. An absolutely connected 3-line $\gamma_{0}^{3} \equiv a_{1} a_{2} a_{3}$ has one circuit, and a 4-line $\gamma_{0}^{4} \equiv a_{1} a_{2} a_{3} a_{4}$ has three. By adding lines $a_{5}, a_{6}, \cdots, a_{n}$ successively, we find that the number 
of circuits in the resulting $n$-line $\gamma_{0}{ }^{n}$ is

$$
P=1+2+3+\cdots+(n-2)=(n-1)(n-2) / 2=D .
$$

This is the maximum number of circuits any connected $n$-line can have.

Now we remove $d$ points of connection from $\gamma_{0}{ }^{n}$. If in the resulting $n$-line some or all of the $d$ points of non-connection are inner points, we transform the $n$-line into another one $\gamma_{d}{ }^{n}$ in which all the $d$ points of non-connection are outer points. It is easy to see that the removal of each point of connection from the original $\gamma_{0^{n}}{ }^{n}$ diminishes the number of circuits by one. Therefore, the number $p$ of circuits in $\gamma_{d}^{n}$ is such that

$$
p+d=P=D=(n-1)(n-2) / 2 .
$$

8. Relations to plane curves. When an $n$-line $\gamma_{d^{n}}$ is given, $n$ and $d$ are assumed known. The elementary enumerative properties of $\gamma_{d^{n}}$ so far obtained are all expressed in terms of $n$ and $d$. By means of (7) $M, I, T$ may be expressed as follows:

$$
\begin{aligned}
M & =n-1+p, \\
I & =n-2+2 p, \\
T & =(n-3)(n-2+2 p) / 2+p(p-1) / 2 .
\end{aligned}
$$

It is of interest to note that these formulas or those expressed in terms of $n$ and $d$ bear a close relation to Plucker's equations connecting the class $m$, the number $i$ of inflexions, the number $t$ of bitangents, and also the genus $p$ of a plane algebraic curve of order $n$ with $d$ nodes and no cusps. Comparing our results with those equations, we find that $m=2 M, i=3 I, t=4 T$, and that the genus of the curve is equal to the number of circuits in $\gamma_{d^{n}}$. At present we are not offering any explanation* for these phenomena.

9. Conclusion. Our results are not entirely new. A number of geometers $\dagger$ have already derived numerous enumerative properties of algebraic curves in $r$-space from those of $n$-lines also in $r$-space. The writer himself has in two papers $\ddagger$ done practically the same thing in

* An explanation may be offered from the point of view of topology but at present we are still in search of a more elementary one.

† Severi, Zeuthen, Tanturri, for example. See C. Segre, Mehrdimensionale Räume, Encyklopädie der mathematischen Wissenschaften, vol. $\mathrm{III}_{2}$, no. 7, pp. 878-890.

$\ddagger$ On loci of $(r-2)$-spaces incident with curves in $r$-space, this Bulletin, vol. 36 (1930), pp. 755-761, and Enumerative properties of $r$-space curves, this Bulletin, vol. 40 (1934), pp. 291-296. 
a different manner, but has not explained sufficiently the method of deriving the enumerative properties of the $n$-lines and that of translating them into those of algebraic curves. The present paper is an attempt to put this method on a firmer basis, attention being confined to plane $n$-lines only.

Again, Severi* has considered $n$-lines for the purpose of classifying algebraic curves, and has also discussed the question as to whether a family of curves of order $n$ defined in a certain manner contains at least one $n$-line as one of its members. Our purpose here has nothing to do with Severi's problems. For us, two $n$-lines, however different they may be according to Severi or from the point of view of collineations, have the same enumerative properties, provided they have the same number of points of non-connection or the same number of circuits, and our purpose is to obtain these properties and incidentally point out their relations to those of curves of order $n$ with $d$ nodes in the plane.

University of CALIFornia

* Severi-Löffler, Vorlesungen über algebraische Geometrie, 1921, Appendix G. 